\title{
Các nhân tố ảnh hưởng đến quyết định duy trì khách hàng tại các Công ty kiểm toán độc lập - nghiên cứu thực nghiệm tại Việt Nam
}

\section{Factors affect on client continuance decisions at audit firms - An empirical study in Vietnam}

\author{
Trần Thị Giang Tân ${ }^{1}$, Trần Thị Thu Phường ${ }^{2 *}$ \\ ${ }^{1}$ Trường Đại học Kinh tế Thành phố Hồ Chí Minh, Việt Nam \\ ${ }^{2}$ Trường Đại học Tài Chính - Kế toán, Việt Nam \\ *Tác giả liên hệ, Email: tranthithuphuong@tckt.edu.vn
}

\section{THÔNG TIN}

DOI: $10.46223 / \mathrm{HCMCOUJS}$. econ.vi.16.1.748.2021

Ngày nhận: 13/08/2020

Ngày nhận lại: 02/10/2020

Duyệt đăng: 06/10/2020

\section{Tù khóa:}

duy trì khách hàng, rủi ro kiểm toán, rủi ro kinh doanh của khách hàng, rủi ro kinh doanh của công ty kiểm toán

Keywords:

client continuance, audit risk, business risk

\section{TÓM TÁT}

Duy trì khách hàng là việc công ty kiểm toán chấp nhận tiếp tục kiểm toán cho công ty khách hàng trong niên độ tiếp theo khi được công ty khách hàng tiếp tục mời kiểm toán. Nghiên cứu này nhằm xem xét các nhân tố ảnh hưởng đến quyết định duy trì khách hàng tại các Công ty kiểm toán độc lập tại Việt Nam. Dựa trên kết quả của nghiên cứu trước, mô hình nghiên cứu được xây dựng và kiểm định tại Việt Nam. Mẫu nghiên cứu bao gồm 762 khách hàng niêm yết trên thị trường chứng khoán Việt Nam tại cả hai Sở Giao dịch Chứng khoán Thành phố Hồ Chí Minh và Hà Nội trong giai đoạn từ 2016 - 2019. Kết quả nghiên cứu cho thấy, mô hình gồm ba nhân tố: Rủi ro kiểm toán, rủi ro tài chính của khách hàng và rủi ro kinh doanh của công ty kiểm toán có tác động ngược chiều đến việc duy trì khách hàng. Rủi ro kiểm toán được xem xét thông qua loại ý kiến kiểm toán (gồm ý kiến không phải là chấp nhận toàn phần, ý kiến có đoạn nhấn mạnh về hoạt động liên tục), tỷ lệ giữa các khoản phải thu và hàng tồn kho trên tổng tài sản, hành vi điều chỉnh lợi nhuận. Rủi ro tài chính của khách hàng được đo lường thông qua hệ số $Z$ Score và rủi ro kinh doanh được đo lường thông qua số lượng kiểm toán viên của các công ty kiểm toán độc lập. Dựa trên kết quả này, chúng tôi đề xuất những yếu tố cần xem xét khi quyết định duy trì khách hàng ở các công ty kiểm toán độc lập Việt Nam.

\section{ABSTRACT}

The research aims to examine factors affecting client continuance decisions at audit firms in Vietnam. Based on the result of previous research, a research model was developed and tested in Vietnam. The data was collected from 762 listed clients in HOSE and HNX during the period year from 2016 to 2019. Our findings show that three factors: the audit risk, the financial risk and the auditor's business risk have negatively associated with client continuance decisions. The audit risk includes consideration of types of opinions (modified opinions, going concern opinions), the client growth, the ratio of account receivables and inventories to 
total assets, accruals-based earnings management as important factors when deciding to continue client and selecting appropriate risk responses. The financial risk is measured through $\mathrm{Z}$ Score and the auditor's business risk is measured through the number of independent auditors. Based on these results, we propose considerations when deciding to continue clients at auditing firms in Vietnam.

\section{Giới thiệu}

Chấp nhận khách hàng mới hay duy trì khách hàng cũ là giai đoạn đầu tiên và rất quan trọng trong quy trình kiểm toán. Bằng cách sàng lọc các khách hàng trước khi ký kết hợp đồng, công ty kiểm toán có thể giảm thiểu các rủi ro như thiệt hại tài chính, sụt giảm uy tín và thậm chí là phải ngừng hoạt động. Để giảm thiểu các rủi ro này, chuẩn mực kiểm toán Việt Nam (VSA 220) yêu cầu các công ty kiểm toán cần phải thiết lập chính sách và thủ tục kiểm soát liên quan đến việc chấp nhận khách hàng mới hoặc tiếp tục khách hàng cũ. Bên cạnh các quy định của chuẩn mực kiểm toán, khá nhiều nghiên cứu trước đã phát hiện các nhân tố ảnh hưởng đến quyết định chấp nhận hay duy trì khách hàng như: Rủi ro kiểm toán, rủi ro kinh doanh của khách hàng và rủi ro kinh doanh của công ty kiểm toán. Ba loại rủi ro này có ảnh hưởng ngược chiều đến quyết định chấp nhận hay duy trì khách hàng (Ebaid, 2011; Hsieh \& Lin, 2016; Johnstone, 2000; Johnstone \& Bedard, 2003; Johnstone \& Bedard, 2004; Ouertani \& DamakAyadi, 2012). Tại Việt Nam, cho đến nay, có rất ít nghiên cứu về các nhân tố ảnh hưởng đến quyết định chấp nhận hay duy trì khách hàng. Một vài nghiên cứu tìm hiểu về rủi ro từ việc không tuân thủ quy trình chấp nhận khách hàng (Nguyen, 2013), đánh giá trọng yếu và rủi ro kiểm toán (Doan, 2011) trong chấp nhận khách hàng. Do vậy, câu hỏi được đặt ra là các nhân tố nào ảnh hưởng đến quyết định duy trì khách hàng tại các Công ty kiểm toán độc lập Việt Nam. Để trả lời cho câu hỏi nghiên cứu này, chúng tôi dựa vào các nghiên cứu trước để thiết lập mô hình, giả thuyết nghiên cứu và kiểm định giả thuyết này nhằm đưa ra gợi ý cho các công ty kiểm toán độc lập tại Việt Nam.

Đóng góp chính trong nghiên cứu của chúng tôi là xác lập mô hình về các nhân tố ảnh hưởng đến việc duy trì khách hàng tại Việt Nam dựa trên dữ liệu các công ty niêm yết trên thị trường chứng khoán. Kết quả nghiên cứu này giúp bổ sung những nội dung cần chú ý khi duy trì khách hàng bên cạnh các hướng dẫn của VSA 220. Ngoài ra, nghiên cứu của chúng tôi cũng làm rõ các nhân tố mà công ty kiểm toán cần quan tâm khi đánh giá từng loại rủi ro. Đối với rủi ro kiểm toán, đó là các loại ý kiến kiểm toán (ý kiến không phải là chấp nhận toàn phần, hoạt động liên tục), mức tăng trưởng của công ty được kiểm toán, tỷ lệ giữa các khoản phải thu và hàng tồn kho trên tổng tài sản, hành vi điều chỉnh lợi nhuận. Đối với rủi ro tài chính, đó là xem xét hệ số Z-Score, còn đối với rủi ro của công ty kiểm toán, đó là khả năng thực hiện kiểm toán. Dựa trên kết quả nghiên cứu này, chúng tôi đề xuất thêm một số kiến nghị khác cho công ty kiểm toán độc lập tại Việt Nam.

Sau phần giới thiệu, nghiên cứu của chúng tôi được cấu trúc thành năm (5) phần chính, phần hai (2) trình bày về cơ sở lý thuyết, phần ba (3) trình bày các nghiên cứu trước về các nhân tố ảnh hưởng đến quyết định chấp nhận hay duy trì khách hàng, phần bốn (4) trình bày phương pháp nghiên cứu, phần năm (5) trình bày kết quả nghiên cứu, cuối cùng là phần sáu (6) trình bày kết luận và gợi ý hướng nghiên cứu tiếp theo. 


\section{Cơ sở lý thuyết}

\section{- Các khái niệm nền tảng}

+ Quyết định duy trì khách hàng là quá trình bắt đầu từ việc đánh giá khách hàng hiện tại để đưa ra quyết định tiếp tục hay từ chối hợp đồng kiểm toán. Việc từ chối này nhằm loại bỏ những khách hàng không phù hợp, thường là những khách hàng được đánh giá là rủi ro cao hoặc không phù hợp với chiến lược của công ty (Johnstone \& Bedard, 2004);

+ Rủi ro kiểm toán là rủi ro mà kiểm toán viên đưa ra ý kiến kiểm toán không phù hợp khi báo cáo tài chính đã được kiểm toán còn sai sót trọng yếu (VSA 200);

+ Rủi ro kinh doanh: Là rủi ro phát sinh từ các điều kiện, sự kiện, tình huống, việc thực hiện hay không thực hiện các hành động có ảnh hưởng đáng kể mà có thể dẫn đến ảnh hưởng bất lợi tới khả năng đạt được mục tiêu và thực hiện được chiến lược kinh doanh của đơn vị, hoặc là rủi ro phát sinh từ việc xác định mục tiêu và chiến lược kinh doanh không phù hợp (VSA 315).

\section{Nghiên cứu trước về các nhân tố ảnh hưởng đến quyết định chấp nhận hay duy trì khách hàng và giả thuyết nghiên cứu}

Kết quả các nghiên cứu trước cho thấy, nhân tố đầu tiên và rất quan trọng ảnh hưởng đến quyết định chấp nhận hay duy trì khách hàng là rủi ro kiểm toán của công ty kiểm toán (Cenker \& Nagy, 2008; Chow, Ho, \& Mo, 2006; Gendron, 2001; Hsieh \& Lin, 2016; Johnstone, 2000; Johnstone \& Bedard, 2003; Johnstone \& Bedard, 2004; Ouertani \& Ayadi, 2012). Johnstone và Bedard (2003) xem xét liệu rủi ro kiểm toán và các chiến lược đối phó rủi ro (cụ thể là việc sử dụng chuyên gia và tính phí cao hơn) của công ty kiểm toán có ảnh hưởng đến quyết định chấp nhận hay duy trì khách hàng không. Kết quả nghiên cứu cho thấy rủi ro kiểm toán của công ty kiểm toán có ảnh hưởng ngược chiều đến quyết định chấp nhận khách hàng. Trong đó, rủi ro kiểm toán gồm cả rủi ro do gian lận và nhầm lẫn. Năm 2004, Johnstone và Bedard tiếp tục nghiên cứu về quyết định chấp nhận và duy trì khách hàng dựa trên thông tin lưu lại trong cơ sở dữ liệu chấp nhận, duy trì khách hàng trong năm 2000-2001 của một công ty kiểm toán lớn. Kết quả nghiên cứu cho thấy, rủi ro kiểm toán của công ty kiểm toán có ảnh hưởng ngược chiều đến quyết định chấp nhận hay duy trì khách hàng. Trong đó, rủi ro kiểm toán được đo lường qua tính hữu hiệu của kiểm soát nội bộ trong việc lập và trình bày báo cáo tài chính và rủi ro về tính chính trực của người quản lý. Nghiên cứu của Hsieh và Lin (2016) về các nhân tố ảnh hưởng đến quyết định chấp nhận hay duy trì khách hàng thông qua sử dụng thông tin từ cơ sở dữ liệu của Tạp chí Kinh tế Đài Loan cũng cho thấy, rủi ro kiểm toán có ảnh hưởng ngược chiều đến quyết định chấp nhận, duy trì khách hàng. Trong đó, rủi ro kiểm toán được đo lường bằng thành phần tổng hợp từ phân tích nhân tố: Ý kiến kiểm toán không phải là ý kiến chấp nhận toàn phần, ý kiến về hoạt động liên tục, mức tăng trưởng của công ty được kiểm toán, tỷ lệ các khoản phải thu và hàng tồn kho trên tổng tài sản, hành vi điều chỉnh lợi nhuận (được đo lường bởi giá trị tuyệt đối của các khoản dồn tích tự định của các khoản thu nhập ước tính, được tính theo mô hình Jones modified).

Dựa vào các nghiên cứu nêu trên, giả thuyết được đặt ra là:

\section{H1: Rủi ro kiểm toán có ảnh hưởng ngược chiều với quyết định duy trì khách hàng}

Kết quả các nghiên cứu trước còn cho thấy rủi ro tài chính của khách hàng là nhân tố kế tiếp ảnh hưởng đến quyết định chấp nhận hay duy trì khách hàng (Hsieh \& Lin, 2016; Johnstone \& Bedard, 2004). Rủi ro tài chính được đo lường thông qua), hệ số âm Z Score (Hsieh \& Lin, 2016).

Dựa vào các nghiên cứu nêu trên, giả thuyết được đặt ra là: 
H2: Rủi ro tài chính của khách hàng có ảnh huởng ngực chiều với quyết định duy trì khách hàng

Rủi ro kinh doanh của công ty kiểm toán có ảnh hưởng ngược chiều đến quyết định chấp nhận, duy trì khách hàng (Chow et al., 2006; Johnstone, 2000; Johnstone \& Bedard, 2003; Johnstone \& Bedard, 2004; Ouertani \& Ayadi, 2012), được đo lường thông qua khả năng của công ty kiểm toán trong việc thực hiện kiểm toán. Khả năng này được xác định thông qua số lượng kiểm toán viên thực hiện kiểm toán (Ouertani \& Ayadi, 2012).

Dựa vào các nghiên cứu nêu trên, giả thuyết được đặt ra là:

H3: Rủi ro kinh doanh của công ty kiểm toán có ảnh hưởng ngược chiều với quyết định duy trì khách hàng

\section{Phương pháp nghiên cứu}

\subsection{Mô hình nghiên cứu}

Dựa trên kết quả nghiên cứu trước (Hsieh \& Lin, 2016; Johnstone \& Bedard, 2004) về các nhân tố ảnh hưởng đến duy trì khách hàng: Rủi ro kiểm toán, rủi ro tài chính và rủi ro kinh doanh của công ty kiểm toán, chúng tôi thiết kế mô hình nghiên cứu để kiểm tra sự ảnh hưởng này tại các Công ty kiểm toán độc lập tại Việt Nam. Mô hình nghiên cứu gồm biến phụ thuộc là quyết định duy trì khách hàng (CONTINUE/DISCONTINUE); 03 biến độc lập là: Rủi ro kiểm toán (AUDR), rủi ro tài chính (FINR), rủi ro kinh doanh của công ty kiểm toán (BUSR). Ngoài ra, chúng tôi còn đưa vào mô hình một số biến kiểm soát:

- Biến FIRMSIZE: Thể hiện quy mô của khách hàng. Biến FIRMSIZE dựa trên nghiên cứu của Hsieh và Lin (2016), Johnstone và Bedard (2004);

- Biến TENURE: Thể hiện số năm được kiểm toán bởi công ty kiểm toán. Biến TENURE dựa trên nghiên cứu của Hsieh và Lin (2016);

- Biến INTERNALAUDIT: Thể hiện công ty khách hàng có bộ phận kiểm toán nội bộ. Biến INTERNALAUDIT dựa trên nghiên cứu của Ouertani và Ayadi (2012).

Mô hình nghiên cứu (hồi quy Binary Logistic) như sau:

CONTINUE/DISCONTINUE $=\beta_{0}+\beta_{1}$ AUDR $+\beta_{2}$ FINR $+\beta_{3}$ BUSR $+\beta_{4}$ FIRMSIZE + $\beta_{5}$ TENURE $+\beta_{6}$ INTERNALAUDIT $+\mathrm{U}_{\mathrm{i}}$

\subsection{Thang đo các biến nghiên cứu}

Cách thức đo lường các biến trong mô hình được trình bày trong Bảng 1 .

\section{Bảng 1}

Tóm tắt các biến

\begin{tabular}{|c|c|c|c|c|}
\hline Mã biến & Tên biến & Đặc tính & Cách thức đo lường & $\begin{array}{c}\text { Dấu kỳ } \\
\text { vọng }\end{array}$ \\
\hline \multicolumn{5}{|l|}{ Biến phụ thuộc } \\
\hline $\begin{array}{c}\text { CONTINUE/ } \\
\text { DISCONTINUE }\end{array}$ & $\begin{array}{l}\text { Quyết định duy trì } \\
\text { khách hàng }\end{array}$ & & $\begin{array}{l}=1 \text { nếu tiếp tục duy trì khách hàng kiểm } \\
\text { toán và }=0 \text { là ngược lại. }\end{array}$ & \\
\hline \multicolumn{5}{|l|}{ Biến độc lập } \\
\hline AUDR & Rủi ro kiểm toán & & $\begin{array}{l}=\text { Thành phần tổng hợp từ phân tích } \\
\text { nhân tố (EFA) của các nhân tố thuộc rủi }\end{array}$ & - \\
\hline
\end{tabular}




\begin{tabular}{|c|c|c|c|c|}
\hline Mã biến & Tên biến & Đặc tính & Cách thức đo lường & $\begin{array}{c}\text { Dấu kỳ } \\
\text { vọng }\end{array}$ \\
\hline & & & $\begin{array}{l}\text { ro kiểm toán: MODOP, GC, GROWTH, } \\
\text { INVREC, ABSDA. }{ }^{1}\end{array}$ & \\
\hline \multicolumn{5}{|l|}{ Trong đó: } \\
\hline MODOP & $\begin{array}{l}\text { Ý kiến kiểm toán } \\
\text { không phải là ý } \\
\text { kiến chấp nhận } \\
\text { toàn phần }\end{array}$ & $\begin{array}{l}\text { Biến định } \\
\text { tính }\end{array}$ & $\begin{array}{l}\text { = } 1 \text { nếu khách hàng nhận ý kiến kiểm } \\
\text { toán khồng phải là ý kiến chấp nhận } \\
\text { toàn phần (trong báo cáo kiểm toán năm } \\
\text { trước) và }=0 \text { trường hợp khác. }\end{array}$ & \\
\hline GC & $\begin{array}{l}\text { Ý kiến về hoạt } \\
\text { động liên tục }\end{array}$ & $\begin{array}{l}\text { Biến định } \\
\text { tính }\end{array}$ & $\begin{array}{l}=1 \text { nếu ý kiến kiểm toán có đoạn nhấn } \\
\text { mạnh về hoạt động liên tục và }=0 \\
\text { trường hợp khác. }\end{array}$ & \\
\hline GROWTH & $\begin{array}{l}\text { Sự tăng trưởng của } \\
\text { công ty được kiểm } \\
\text { toán }\end{array}$ & $\begin{array}{l}\text { Biến định } \\
\text { lượng }\end{array}$ & $\begin{array}{l}=\text { (Tổng tài sản cuối năm - Tổng tài sản } \\
\text { đầu năm)/Tồng tài sản đầu năm. }\end{array}$ & \\
\hline INVREC & $\begin{array}{l}\text { Tỷ lệ các khoản } \\
\text { phải thu và hàng } \\
\text { tồn kho trên tồng } \\
\text { tài sản }\end{array}$ & $\begin{array}{l}\text { Biến định } \\
\text { lượng }\end{array}$ & $\begin{array}{l}=\text { Các khoản phải thu và hàng tồn kho } \\
\text { trên tổng tài sản. }\end{array}$ & \\
\hline ABSDA & $\begin{array}{l}\text { Hành vi điều chỉnh } \\
\text { lợi nhuận }\end{array}$ & $\begin{array}{l}\text { Biến định } \\
\text { lượng }\end{array}$ &  & \\
\hline FINR & Rủi ro tài chính & $\begin{array}{l}\text { Biến định } \\
\text { lượng }\end{array}$ & $\begin{array}{l}=- \text { Zscore. } \\
\text { Trong đó: Zscore = } 1.51(\mathrm{CA}-\mathrm{CL}) / \mathrm{TA}+ \\
\text { 1.0(RE /TA }+ \text { 6.2EBITA + } \\
0.1(\mathrm{MVEQ} / \mathrm{TL})+1.7(\mathrm{SALES} / \mathrm{TA}) \text {. } \\
\text { Trong đó CA: là tài sản ngắn hạn; CL: } \\
\text { nợ ngắn hạn; TA: tổng tài sản; RE: thu }\end{array}$ & - \\
\hline
\end{tabular}

\footnotetext{
${ }^{1}$ Hệ số tải của các nhân tố MODOP, GC, GROWTH, INVREC, ABSDA tương ứng là 0,$784 ; 0,778 ; 0,789 ; 0,744$; 0,952 . Các nhân tố đều có hệ số tải nhân tố (Factor loading values) > 0,3. Các nhân tố này đều được giữ lại. Phân tích EFA trích được 3 nhóm nhân tố với Eigenvalues $>1$. Chúng tôi sử dụng thành phần chính thứ nhất - the first principal component (có eigenvalue lớn nhất) làm đại diện cho nhóm các nhân tố này.
} 


\begin{tabular}{|c|c|c|c|c|}
\hline Mã biến & Tên biến & Đặc tính & Cách thức đo lường & $\begin{array}{l}\text { Dấu kỳ } \\
\text { vọng }\end{array}$ \\
\hline & & & $\begin{array}{l}\text { nhập giữ lại; EBITA: (lợi nhuận trước } \\
\text { thuế và lãi vay)/tổng tài sản; MVEQ: } \\
\text { giá thị trường của vốn chủ sở hữu; TL: } \\
\text { tổng nợ phải trả và SALES: tổng doanh } \\
\text { số. }\end{array}$ & \\
\hline BUSR & $\begin{array}{l}\text { Rủi ro kinh doanh } \\
\text { của công ty kiểm } \\
\text { toán }\end{array}$ & $\begin{array}{l}\text { Biến định } \\
\text { lượng }\end{array}$ & $\begin{array}{l}\text { Rủi ro kinh doanh công ty kiểm toán } \\
\text { được đo lường thông qua khả năng của } \\
\text { công ty kiểm toán trong việc thực hiện } \\
\text { kiểm toán cho các công ty niêm yết. } \\
\text { Trong đó, khả năng của công ty kiểm } \\
\text { toán trong thực hiện kiềm toán cho các } \\
\text { công ty niểm yết đo lường thông qua số } \\
\text { lượng kiểm toán viên được chấp thuận } \\
\text { thực hiện kiểm toán cho đơn vị có lợi } \\
\text { ích công chúng. }\end{array}$ & - \\
\hline \multicolumn{5}{|l|}{ Biến kiểm soát } \\
\hline FIRMSIZE & $\begin{array}{l}\text { Quy mô của khách } \\
\text { hàng }\end{array}$ & $\begin{array}{l}\text { Biến định } \\
\text { lượng }\end{array}$ & $\begin{array}{l}=\text { Logarit tự nhiên của tổng doanh thu } \\
\text { vào cuối năm. }\end{array}$ & + \\
\hline TENURE & $\begin{array}{l}\text { Số năm được kiểm } \\
\text { toán bởi công ty } \\
\text { kiểm toán }\end{array}$ & $\begin{array}{l}\text { Biến định } \\
\text { lượng }\end{array}$ & $\begin{array}{l}\text { = Số năm được kiểm toán bởi công ty } \\
\text { kiểm toán trong năm trước. }\end{array}$ & - \\
\hline INTERNALAUDIT & $\begin{array}{l}\text { Bộ phận kiểm toán } \\
\text { nội bộ }\end{array}$ & $\begin{array}{l}\text { Biến định } \\
\text { tính }\end{array}$ & $\begin{array}{l}=1 \text { nếu Công ty có bộ phận kiểm toán } \\
\text { nội bộ và = } 0 \text { trường hợp khác. }\end{array}$ & + \\
\hline
\end{tabular}

Nguồn: Hsieh và Lin (2016); Johnstone và Bedard (2004)

\subsection{Mẫu nghiên cứu}

Dữ liệu nghiên cứu được thu thập từ báo cáo tài chính, báo cáo thường niên, báo cáo quản trị, website của công ty niêm yết, cafef.vn hay các trang web khác.

Mẫu nghiên cứu sơ bộ bao gồm 762 khách hàng niêm yết trên thị trường chứng khoán Việt Nam tại cả hai Sở Giao dịch Chứng khoán Thành phố Hồ Chí Minh và Hà Nội được kiểm toán bởi các Công ty kiểm toán độc lập với tổng số 2.934 quan sát trong giai đoạn từ 2016 - 2019.

Sau khi loại bỏ các quan sát liên quan đến khách hàng là các công ty tài chính và các quan sát không có đủ dữ liệu, mẫu nghiên cứu cuối cùng của chúng tôi gồm 2.478 quan sát.

\section{Kết quả nghiên cứu}

Để kiểm định ảnh hưởng của các nhân tố trên đến quyết định duy trì khách hàng, chúng tôi sử dụng phần mềm SPSS 22.

\subsection{Thống kê mô tả các biến}

Bảng 2 bên dưới trình bày phần thống kê mô tả các biến trong mô hình nghiên cứu. Kết quả các biến trong quyết định duy trì khách hàng tại các Công ty kiểm toán độc lập Việt Nam như sau:

- CONTINUE/DISCONTINUE có giá trị trung bình là 0,813 , điều này có nghĩa là tỷ lệ trung bình quyết định duy trì khách hàng là $81,3 \%$, hay nói khác hơn, 18,7\% trường hợp công ty 
kiểm toán từ chối khách hàng;

- AUDR có giá trị trung bình là $-0,005$, điều này có nghĩa là rủi ro kiểm toán trung bình là $-0,005$;

- FINR có giá trị trung bình là -3,201, điều này có nghĩa là hệ số phá sản (Z-Score) trung bình của các doanh nghiệp niêm yết trên thị trường chứng khoán Việt Nam là 3,201;

- BUSR có giá trị trung bình là 31,688 , điều này có nghĩa là số lượng kiểm toán viên được chấp thuận thực hiện kiểm toán cho đơn vị có lợi ích công chúng trung bình của mỗi công ty kiểm toán khoảng 31,688 người;

- FIRMSIZE có giá trị trung bình là 27,084, điều này có nghĩa là logarit tự nhiên của tổng doanh thu của khách hàng trung bình là 27,08398;

- TENURE có giá trị trung bình là 4,660, điều này có nghĩa là số năm được kiểm toán bởi công ty kiểm toán trong năm trước trung bình là 4,660 năm;

- INTERNALAUDIT có giá trị trung bình là 0,105 , điều này có nghĩa là tỷ lệ trung bình công ty khách hàng có bộ phận kiểm toán nội bộ là $10,5 \%$.

\section{Bảng 2}

Bảng thống kê mô tả các biến hồi quy

\begin{tabular}{|l|r|r|r|r|r|}
\hline & $\begin{array}{c}\text { Số quan sát } \\
\text { N }\end{array}$ & $\begin{array}{c}\text { Giá trị nhỏ } \\
\text { nhất }\end{array}$ & $\begin{array}{c}\text { Giá trị lớn } \\
\text { nhất }\end{array}$ & $\begin{array}{c}\text { Giá trị } \\
\text { trung bình }\end{array}$ & $\begin{array}{c}\text { Độ lệch } \\
\text { chuẩn }\end{array}$ \\
\hline CONTINUE/DISCONTINUE & 2478 & 0 & 1 & 0,813 & 0,390 \\
AUDR & 2478 & $-1,192$ & 31,686 & $-0,005$ & 0,970 \\
FINR & 2478 & $-27,603$ & 5,205 & $-3,201$ & 2,588 \\
BUSR & 2478 & 15 & 57 & 31,688 & 14,386 \\
FIRMSIZE & 2478 & 18,729 & 32,876 & 27,084 & 1,649 \\
TENURE & 2478 & 1 & 12 & 4,660 & 2,938 \\
INTERNALAUDIT & 2478 & 0 & 1 & 0,105 & 0,3070 \\
Valid N (listwise) & 2478 & & & & \\
\hline
\end{tabular}

Nguồn: Từ SPSS 22

Bảng 3 trình bày phần thống kê mô tả các yếu tố thuộc rủi ro kiểm toán (AUDR). Kết quả cho thấy trong các quyết định duy trì khách hàng tại các Công ty kiểm toán độc lập Việt Nam:

- MODOP có giá trị trung bình là 0,060 , điều này có nghĩa là tỷ lệ trung bình báo cáo kiểm toán có ý kiến kiểm toán không phải là ý kiến chấp nhận toàn phần là $6 \%$;

- GC có giá trị trung bình là 0,114 , điều này có nghĩa là tỷ lệ trung bình báo cáo kiểm toán có ý kiến về hoạt động liên tục là $11,4 \%$;

- GROWTH có giá trị trung bình là 0,134 , điều này có nghĩa là tỷ lệ trung bình chênh lệch giữa tổng tài sản cuối năm - tổng tài sản đầu năm trên tổng tài sản đầu năm là $13,4 \%$;

- INVREC có giá trị trung bình là 0,471 , điều này có nghĩa là tỷ lệ trung bình giữa các khoản phải thu và hàng tồn kho trên tổng tài sản là $47,1 \%$;

- ABSDA có giá trị trung bình là 0,15 , điều này có nghĩa là mức điều chỉnh lợi nhuận 
trung bình tại Việt Nam là $15 \%$.

\section{Bảng 3}

Bảng thống kê mô tả các yếu tố thuộc rủi ro kiểm toán (AUDR)

\begin{tabular}{|l|r|r|r|r|r|}
\hline & Số quan sát $\mathbf{~}$ & \multicolumn{1}{|c|}{$\begin{array}{c}\text { Giá trị nhỏ } \\
\text { nhất }\end{array}$} & $\begin{array}{c}\text { Giá trị lớn } \\
\text { nhất }\end{array}$ & $\begin{array}{c}\text { Giá trị trung } \\
\text { bình }\end{array}$ & $\begin{array}{c}\text { Độ lệch } \\
\text { chuẩn }\end{array}$ \\
\hline MODOP & 2478 & 0,0 & 1,0 & 0,060 & 0,237 \\
GC & 2478 & 0,0 & 1,0 & 0,114 & 0,318 \\
GROWTH & 2478 & $-0,842$ & 21,193 & 0,134 & 0,669 \\
INVREC & 2478 & 0,004 & 0,991 & 0,471 & 0,249 \\
ABSDA & 2478 & 0,0001 & 4,653 & 0,150 & 0,162 \\
Valid N (listwise) & 2478 & & & & \\
\hline
\end{tabular}

Nguồn: Từ SPSS 22

\subsection{Phân tích tương quan giũa các biến}

Thực hiện bước này là nhằm xem xét mối quan hệ tương quan giữa các biến các biến độc lập và biến phụ thuộc thông qua phân tích tương quan Pearson. Kết quả ở Bảng 4 cho thấy, các biến độc lập như rủi ro kiểm toán (AUDR), rủi ro tài chính (FINR), rủi ro kinh doanh của công ty kiểm toán (BUSR), số năm kiểm toán (TENURE), quy mô của khách hàng (FIRMSIZE) đều có giá trị Sig. $<0,05$. Điều đó chứng tỏ các nhân tố này đều có ý nghĩa thống kê, tức các biến này đều phù hợp đưa vào mô hình nghiên cứu. Tuy nhiên, biến kiểm toán nội bộ (INTERNALAUDIT) có giá trị Sig. > 0,05 chứng tỏ các biến này không có ý nghĩa thống kê nên bị loại trừ khi phân tích hồi quy. Mặt khác, hệ số tương quan tuyệt đối giữa các biến đều nằm trong giới hạn từ 0,003 đến $0,257 \leq 0,8$, như vậy, mô hình nghiên cứu không xảy ra hiện tượng đa cộng tuyến. 


\section{Bảng 4}

Bảng phân tích tương quan Pearson

\begin{tabular}{|c|c|c|c|c|c|c|c|c|}
\hline & & CONTINUE/DISCONTINUE & AUDR & FINR & BUSR & FIRM SIZE & TENURE & INTERNALAUDIT \\
\hline CONTINUE/ & Tương quan Pearson & 1 & $-0,098^{* *}$ & $-0,118^{* *}$ & $0,138^{* *}$ & $0,119^{* *}$ & $0,170^{* *}$ & 0,006 \\
\hline \multirow[t]{2}{*}{ DISCONTINUE } & Sig. (2 nhân tố) & & 0,000 & 0,000 & 0,000 & 0,000 & 0,000 & 0,754 \\
\hline & Số quan sát (N) & 2478 & 2478 & 2478 & 2478 & 2478 & 2478 & 2478 \\
\hline \multirow[t]{3}{*}{ AUDR } & Tương quan Pearson & $-0,098^{* *}$ & 1 & 0,006 & $-0,073^{* *}$ & $-0,016$ & $-0,059^{* *}$ & 0,003 \\
\hline & Sig. (2 nhân tố) & 0,000 & & 0,779 & 0,000 & 0,438 & 0,003 & 0,894 \\
\hline & Số quan sát (N) & 2478 & 2478 & 2478 & 2478 & 2478 & 2478 & 2478 \\
\hline \multirow[t]{3}{*}{ FINR } & Tương quan Pearson & $-0,118^{* *}$ & 0,006 & 1 & $-0,041^{*}$ & $-0,149^{* *}$ & $-0,026$ & $0,041^{*}$ \\
\hline & Sig. (2 nhân tố) & 0,000 & 0,779 & & 0,039 & 0,000 & 0,200 & 0,040 \\
\hline & Số quan sát (N) & 2478 & 2478 & 2478 & 2478 & 2478 & 2478 & 2478 \\
\hline \multirow[t]{3}{*}{ BUSR } & Tương quan Pearson & $0,138^{* *}$ & $-0,073^{* *}$ & $-0,041^{*}$ & 1 & $0,243^{* *}$ & $0,257^{* *}$ & $0,094^{* *}$ \\
\hline & Sig. (2 nhân tố) & 0,000 & 0,000 & 0,039 & & 0,000 & 0,000 & 0,000 \\
\hline & Số quan sát (N) & 2478 & 2478 & 2478 & 2478 & 2478 & 2478 & 2478 \\
\hline \multirow[t]{3}{*}{ FIRMSIZE } & Tương quan Pearson & $0,119^{* *}$ & $-0,016$ & $-0,149^{* *}$ & $0,243^{* *}$ & 1 & $0,074^{* *}$ & $0,154^{* *}$ \\
\hline & Sig. (2 nhân tố) & 0,000 & 0,438 & 0,000 & 0,000 & & 0,000 & 0,000 \\
\hline & Số quan sát (N) & 2478 & 2478 & 2478 & 2478 & 2478 & 2478 & 2478 \\
\hline \multirow[t]{3}{*}{ TENURE } & Tương quan Pearson & $0,170^{* *}$ & $-0,059^{* *}$ & $-0,026$ & $0,257^{* *}$ & $0,074^{* *}$ & 1 & $0,040^{*}$ \\
\hline & Sig. (2 nhân tố) & 0,000 & 0,003 & 0,200 & 0,000 & 0,000 & & 0,046 \\
\hline & Số quan sát (N) & 2478 & 2478 & 2478 & 2478 & 2478 & 2478 & 2478 \\
\hline \multirow[t]{3}{*}{ INTERNALAUDIT } & Tương quan Pearson & 0,006 & 0,003 & $0,041^{*}$ & $0,094^{* *}$ & $0,154^{* *}$ & $0,040^{*}$ & 1 \\
\hline & Sig. (2 nhân tố) & 0,754 & 0,894 & 0,040 & 0,000 & 0,000 & 0,046 & \\
\hline & Số quan sát (N) & 2478 & 2478 & 2478 & 2478 & 2478 & 2478 & 2478 \\
\hline
\end{tabular}

**. Tương quan có ý nghĩa thống kê tại mức 0,01 (phân tích mối tương quan 2 nhân tố)

*. Tương quan có ý nghĩa thống kê tại mức 0,05 (phân tích mối tương quan 2 nhân tố)

Nguồn: Từ SPSS 22 
Thông qua phân tích hệ số tương quan (Bảng 4), có thể thấy, hệ số tương quan Pearson giữa các biến có giá trị nhỏ, có nghĩa không xảy ra hiện tượng đa cộng tuyến giữa các biến độc lập.

\subsection{Phân tích hồi quy Binary Logistic}

Để kiểm định các giả thuyết nghiên cứu, chúng tôi tiến hành phân tích hồi quy Binary Logistic.

\section{- Mức độ phù hợp của mô hình:}

Bảng 5 thể hiện kết quả của kiểm định Wald (kiểm định giả thuyết hồi quy khác không). Kết quả kiểm tra với các hệ số $\beta$ ở Bảng 5 cho thấy, ba giá trị sig đều $=0,000<0,05$ nên có thể kết luận các hệ số $\beta$ trong mô hình là khác không (0). Như vậy, các hệ số hồi quy có ý nghĩa, mô hình nghiên cứu phù hợp.

\section{Bảng 5}

Kết quả kiểm tra đối với các hệ số $\beta$

Omnibus Tests of Model Coefficients

\begin{tabular}{|ll|c|c|c|}
\hline & Chi-square & df & Sig. \\
\hline Step 1 & Step & 165,487 & 5 & 0,000 \\
& Block & 165,487 & 5 & 0,000 \\
& Model & 165,487 & 5 & 0,000 \\
\hline
\end{tabular}

Nguồn: Từ SPSS 22

\section{- Mức độ giải thích của mô hình:}

Bảng 6 cho thấy hệ số hiệu chỉnh $\mathrm{R}^{2}=0,245$. Kết quả này cho thấy các biến trong mô hình có thể giải thích được $24,5 \%$ quyết định duy trì khách hàng tại các Công ty kiểm toán độc lập Việt Nam.

\section{Bảng 6}

Kết quả tóm tắt của mô hình

\begin{tabular}{|c|c|c|c|}
\hline Bước & Thông số -2LL & Hệ số $\mathbf{R}^{\mathbf{2}}$ giả & Hệ số hiệu chỉnh $\mathbf{R}^{\mathbf{2}}$ \\
\hline 1 & $460,668^{\mathrm{a}}$ & 0,160 & 0,245 \\
\hline
\end{tabular}

a. Ước tính kết thúc ở lần lặp số 5 vì ước tính tham số thay đổi ít hơn 0,001

Nguồn: Từ SPSS 22

\section{- Kiểm định ý nghĩa các hệ số hồi quy:}

Kết quả phân tích hồi quy Binary Logistic (Bảng 7) cho thấy rủi ro kiểm toán (được đo lường bằng thành phần tổng hợp từ phân tích nhân tố của các yếu tố thuộc rủi ro kiểm toán gồm MODOP, GC, GROWTH, INVREC, ABSDA), rủi ro tài chính (được đo lường thông qua hệ số âm $\mathrm{Z}$ Score) và rủi ro kinh doanh của công ty kiểm toán (được đo lường thông qua số lượng kiểm toán viên được chấp thuận thực hiện kiểm toán cho đơn vị có lợi ích công chúng) có ảnh hưởng ngược chiều đến quyết định duy trì khách hàng với giá trị Sig. $<0.05$. Vì vậy, giả thuyết H1, H2, H3 được chấp nhận.

Mặt khác, kết quả phân tích hồi quy Logistic cho thấy các biến kiểm soát còn lại đều có ý nghĩa thống kê, với giá trị Sig. $<0,05$ và Sig. $<0,1$. Điều này cho thấy số năm được kiểm toán 
bởi công ty kiểm toán (TENURE), quy mô của khách hàng (FIRMSIZE) ảnh hưởng cùng chiều đến quyết định duy trì khách hàng.

\section{Bảng 7}

Kết quả phân tích mô hình hồi quy Binary Logistic

Variables in the Equation

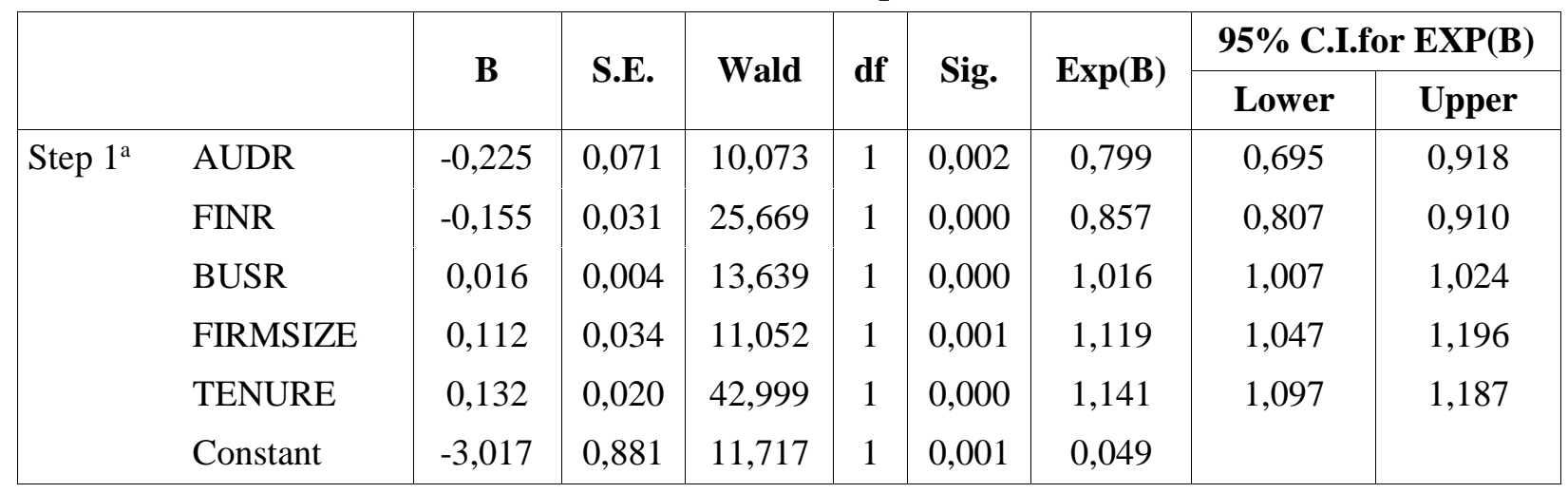

a. Variable(s) entered on step 1: AUDR, FINR, BUSR, FIRMSIZE, TENURE

Nguồn: Từ SPSS 22

Kết quả này chính là cơ sở để chúng tôi đề xuất các kiến nghị nhằm giúp các Công ty kiểm toán độc lập tại Việt Nam đưa ra quyết định duy trì khách hàng phù hợp.

\section{- Thảo luận kết quả hồi quy}

+ Phương trình hồi quy Logistic: Từ kết quả hồi quy ở trên (Bảng 7), ta có phương trình Logistic như sau:

$$
\begin{aligned}
\log _{\mathrm{e}}\left[\mathrm{P}_{0} /\left(1-\mathrm{P}_{0}\right)\right]= & -3,017-0,225 \mathrm{AUDR}-0,155 \mathrm{FINR}+0,016 \mathrm{BUSR} \\
& +0,112 \text { FIRMSIZE }+ \text { 0,132TENURE }
\end{aligned}
$$

+ Ý nghĩa các hệ số hồi quy:

- Hệ số hồi quy của biến rủi ro kiểm toán là $-0,225$. Nếu rủi ro kiểm toán tăng thêm 1 với điều kiện các yếu tố khác không đổi, thì Log của tỷ lệ xác suất quyết định duy trì khách hàng và xác suất không duy trì khách hàng giảm 0,225 lần.

Giải thích theo phương trình gốc của hàm Logit không rõ nghĩa trong kinh tế. Agresti (2007) đưa ra cách giải thích rõ hơn trong kinh tế như sau:

Đặt $\mathrm{P}_{0}$ : Xác suất ban đầu; $\mathrm{P}_{1}$ : Xác suất thay đổi. $\mathrm{P}_{1}$ được tính theo công thức sau:

$$
\mathrm{P}_{1}=\frac{\mathrm{P}_{0} \mathrm{xe}^{\mathrm{B}}}{1-\mathrm{P}_{0}\left(1-\mathrm{e}^{\mathrm{B}}\right)}
$$

Trong đó: $\mathrm{e}^{\mathrm{B}}$ : Hệ số tác động của biến.

- Biến rủi ro kiểm toán (AUDR): Có $\mathrm{B}_{1}=-0,225, \mathrm{P}_{0}=10 \%$ và $\mathrm{e}^{\mathrm{B}}=0,799=>\mathrm{P}_{1}=8,2 \%$

Nếu xác suất quyết định duy trì khách hàng ban đầu $\left(\mathrm{P}_{0}\right)$ là $10 \%$, khi các yếu tố khác không đổi, nếu rủi ro kiểm toán tăng thêm 1 , xác suất công ty kiểm toán duy trì khách hàng sẽ là $8,2 \%$ (giảm $1,8 \%$ so với xác suất ban đầu là $10 \%$ ).

- Biến rủi ro tài chính (FINR): Có $\mathrm{B}_{2}=-0,155, \mathrm{P}_{0}=10 \%$ và $\mathrm{e}^{\mathrm{B}}=0,857=>\mathrm{P}_{1}=8,7 \%$ 
Nếu xác suất quyết định duy trì khách hàng ban đầu $\left(\mathrm{P}_{0}\right)$ là $10 \%$, khi các yếu tố khác không đổi, nếu rủi ro tài chính tăng thêm 1 , xác suất công ty kiểm toán duy trì khách hàng sẽ là $8,7 \%$ (giảm $1,3 \%$ so với xác suất ban đầu là $10 \%$ ).

- Biến rủi ro kinh doanh của công ty kiểm toán (BUSR): Có $\mathrm{B}_{3}=0,016, \mathrm{P}_{0}=10 \%$ và $\mathrm{e}^{\mathrm{B}}$ $=1,016=>P_{1}=10,1 \%$

Nếu xác suất quyết định duy trì khách hàng ban đầu $\left(\mathrm{P}_{0}\right)$ là $10 \%$, khi các yếu tố khác không đổi, nếu số lượng kiểm toán viên được chấp thuận thực hiện kiểm toán cho đơn vị có lợi ích công chúng của công ty kiểm toán trong năm tăng thêm 1 , xác suất công ty kiểm toán duy trì khách hàng sẽ là $10,1 \%$ (tăng $0,1 \%$ so với xác suất ban đầu là $10 \%$ ).

$\mathrm{P}_{1}=11,1 \%$

- Biến quy mô của khách hàng (FIRMSIZE): Có $\mathrm{B}_{4}=0,112, \mathrm{P}_{0}=10 \%$ và $\mathrm{e}^{\mathrm{B}}=1,119=>$

Nếu xác suất quyết định duy trì khách hàng ban đầu $\left(\mathrm{P}_{0}\right)$ là $10 \%$, khi các yếu tố khác không đổi, nếu Logarit tự nhiên của tổng doanh thu vào cuối năm tăng thêm 1 , xác suất công ty kiểm toán duy trì khách hàng sẽ là $11,1 \%$ (tăng $1,1 \%$ so với xác suất ban đầu là $10 \%$ ).

- Biến nhiệm kỳ của công ty kiểm toán (TENURE): Số năm được kiểm toán bởi công ty kiểm toán trong năm trước. Có $\mathrm{B}_{5}=0,132, \mathrm{P}_{0}=10 \%$ và $\mathrm{e}^{\mathrm{B}}=1,141=>\mathrm{P}_{1}=11,3 \%$

Nếu xác suất quyết định duy trì khách hàng ban đầu $\left(\mathrm{P}_{0}\right)$ là $10 \%$, khi các yếu tố khác không đổi, nếu số năm được kiểm toán bởi công ty kiểm toán trong năm trước tăng thêm 1 , xác suất công ty kiểm toán duy trì khách hàng sẽ là $11,3 \%$ (tăng $1,3 \%$ so với xác suất ban đầu là $10 \%$ ).

Trong các biến ảnh hưởng đến quyết định duy trì khách hàng, rủi ro kiểm toán (AUDR) có ảnh hưởng mạnh nhất và còn lại theo thứ tự là: Rủi ro tài chính (FINR), nhiệm kỳ của công ty kiểm toán (TENURE), quy mô của khách hàng (FIRMSIZE), rủi ro kinh doanh của công ty kiểm toán (BUSR).

\section{- Dự báo của mô hình hồi quy Logistic:}

Theo Agresti (2007), dạng dự báo của mô hình:

$$
\mathrm{E}\left(\mathrm{Y} / \mathrm{X}_{\mathrm{i}}\right)=\frac{\mathrm{e}^{\mathrm{LnOdds}}}{1+\mathrm{e}^{\mathrm{LnOdds}}}
$$

$\mathrm{E}\left(\mathrm{Y} / \mathrm{X}_{\mathrm{i}}\right)$ : Xác suất để $\mathrm{Y}=1$ xuất hiện khi biến độc lập $\mathrm{X}$ có giá trị cụ thể $\mathrm{X}_{\mathrm{i}}$.

Từ kết quả hồi quy ở trên (Bảng 7):

$$
=>\mathrm{E}(\mathrm{Y} / \mathrm{X})=\frac{\mathrm{e}^{(-3,017-0,225 \text { AUDR }-0,155 \text { FINR }+0,016 \mathrm{BUSR}+0,112 \text { FIRMSIZE }+0,132 \text { TENURE })}}{1+\mathrm{e}^{(-3,017-0,225 \text { AUDR }-0,155 \text { FINR + 0,016BUSR + 0,112FIRMSIZE + 0,132TENURE })}}
$$

Phương trình (5) dự báo khả năng duy trì khách hàng khi các biến độc lập có giá trị cụ thể.

\section{Kết luận và gọii ý hướng nghiên cứu tiếp theo}

\subsection{Kết luận}

Mục đích của nghiên cứu này là nhằm xem xét các nhân tố ảnh hưởng đến quyết định duy trì khách hàng tại các Công ty kiểm toán độc lập Việt Nam. Dựa trên mẫu gồm 762 khách hàng niêm yết trên Sở Giao dịch Chứng khoán Thành phố Hồ Chí Minh và Hà Nội được kiểm toán bởi các Công ty kiểm toán độc lập (với 2.478 quan sát) trong giai đoạn từ 2016 - 2019, chúng tôi đã xây dựng mô hình về các nhân tố ảnh hưởng đến việc duy trì khách hàng tại các 
công ty kiểm toán độc lập tại Việt Nam. Mô hình bao gồm ba biến độc lập có ảnh hưởng đến việc duy trì khách hàng (CONTINUE/DISCONTINUE) theo thứ tự gồm có: (1) biến rủi ro kiểm toán (AUDR) được đo lường bằng thành phần tổng hợp từ phân tích nhân tố của các yếu tố: Ý kiến kiểm toán không phải là ý kiến chấp nhận toàn phần (MODOP), ý kiến về hoạt động liên tục (GC), mức tăng trưởng của công ty được kiểm toán (GROWTH), tỷ lệ các khoản phải thu và hàng tồn kho trên tổng tài sản (INVREC), hành vi điều chỉnh lợi nhuận (ABSDA); (2) biến rủi ro tài chính được đo lường thông qua hệ số âm $\mathrm{Z}$ Score và (3) biến rủi ro kinh doanh của công ty kiểm toán được đo lường thông qua số lượng kiểm toán viên được chấp thuận thực hiện kiểm toán cho đơn vị có lợi ích công chúng. Các biến kiểm soát cũng có có ảnh hưởng đến quyết định duy trì khách hàng theo thứ tự là: (1) số năm được kiểm toán bởi công ty kiểm toán (TENURE); (2) quy mô của khách hàng (FIRMSIZE).

Kết quả nghiên cứu của chúng tôi hoàn toàn tương đồng với các nghiên cứu trước như nghiên cứu của Hsieh và Lin (2016), Ouertani và Ayadi (2012), Johnstone và Bedard (2004), theo đó, các nhân tố nêu trên đều có ảnh hưởng, trong đó rủi ro kiểm toán là nhân tố quan trọng nhất ảnh hưởng đến quyết định duy trì khách hàng. Tuy nhiên, trong nghiên cứu của chúng tôi, nhân tố rủi ro kinh doanh của công ty kiểm toán được đo lường thông qua số lượng kiểm toán viên của các công ty kiểm toán độc lập tương tự như nghiên cứu của Ouertani và Ayadi (2012). Trong một số nghiên cứu trước khác, rủi ro kinh doanh của công ty kiểm toán được đo lường thông qua việc khách hàng có niêm yết hay không (Johnstone, 2000; Johnstone \& Bedard, 2003; Johnstone \& Bedard, 2004).

Dựa trên kết quả nghiên cứu, chúng tôi có một số đề xuất sau đối với công ty kiểm toán:

Quyết định liệu có duy trì khách hàng hay không là một nội dung quan trong trong quy trình kiểm toán, cũng là một trong những yêu cầu của chuẩn mực kiểm toán VSQC1, VSA 220. Tuy nhiên, VSA 220 chỉ yêu cầu xem xét tính chính trực của các chủ sở hữu chính, thành viên chủ chốt của Ban Giám đốc và Ban quản trị khi công ty kiểm toán đưa ra quyết định duy trì khách hàng. Dựa trên kết quả nghiên cứu, chúng tôi đề xuất thêm là để quyết định duy trì khách hàng, đầu tiên, các công ty kiểm toán cần xem xét các yếu tố thuộc rủi ro kiểm toán như ý kiến kiểm toán năm trước có phải là không chấp nhận toàn phần hay có ý kiến về hoạt động liên tục, mức tăng trưởng của công ty được kiểm toán, tỷ lệ các khoản phải thu và hàng tồn kho trên tổng tài sản và hành vi điều chỉnh lợi nhuận để đưa ra quyết định phù hợp. Kế tiếp, công ty kiểm toán cần xem xét hệ số khả năng phá sản của khách hàng. Cuối cùng, cần xem xét khả năng kiểm toán cho công ty này.

Một cách chi tiết hơn, nếu ý kiến trên báo cáo kiểm toán của năm trước không phải chấp nhận toàn phần, hoặc có đoạn nhấn mạnh về hoạt động liên tục, công ty kiểm toán cần hết sức cân nhắc trong việc duy trì khách hàng này. Kế tiếp, cần xem xét khả năng tăng trưởng khách hàng thông qua các chỉ số như tỷ lệ phải thu và hàng tồn kho trên tổng tài sản, nếu tỷ số này cao hơn các công ty cùng ngành là một chỉ dẫn rủi ro cao. Ngoài ra, cần xem xét yếu tố về rủi ro gian lận (như giá trị tuyệt đối của các khoản dồn tích tự định của các khoản thu nhập ước tính lớn), cũng như các chỉ dẫn về khả năng phá sản (thông qua hệ số Z Score) của khách hàng. Nếu rủi ro tổng thể là cao, cộng với khách hàng là công ty niêm yết, mà số lượng kiểm toán viên của công ty không nhiều, công ty kiểm toán không nên chấp nhận khách hàng này khi mà chưa có chiến lược đối phó rủi ro phù hợp. Chiến lược đối phó rủi ro thường là phải tính phí cao hơn để công ty kiểm toán có thể sử dụng chuyên gia, tăng số lượng bằng chứng kiểm toán cần thu thập, cử các kiểm toán viên có kinh nghiệm tham gia nhóm kiểm toán.

Trong nền kinh tế thị trường, các công ty kiểm toán cũng chịu sự cạnh tranh gay gắt như 
các ngành nghề khác. Việc duy trì được khách hàng là một vấn đề không đơn giản, do vậy, nhiều công ty kiểm toán đã phải chấp nhận khách hàng bằng mọi giá. Quyết định như vậy trước mắt có thể giúp công ty kiểm toán tăng doanh thu, tuy nhiên, về lâu dài, công ty kiểm toán sẽ đối mặt với các rủi ro như bị kiện tụng, mất uy tín và dĩ nhiên sẽ tốn nhiều chi phí. Vì vậy, việc xem xét các nhân tố nêu trên khi quyết định chấp nhận khách hàng là vô cùng quan trọng nhằm hạn chế rủi ro cho công ty kiểm toán.

\subsection{Hạn chế và hướng nghiên cứu tiếp theo}

Hạn chế chính trong nghiên cứu là do các quy định hiện hành tại Việt Nam không bắt buộc các công ty niêm yết phải công khai giá phí kiểm toán. Do vậy, chúng tôi không thể thu thập dữ liệu này từ báo cáo tài chính, báo cáo thường niên, báo cáo quản trị, website của công ty niêm yết, cafef.vn hay các trang web khác để đưa vào mô hình. Điều này đưa đến mức độ giải thích của mô hình của chúng tôi chỉ là 0.245 (Nagelkerke $\mathrm{R} 2=0,245$ ). Trong tương lai, khi có yêu cầu bắt buộc phải công bố các thông tin liên quan đến kiểm toán một cách rộng rãi để có thể thu thập dữ liệu cho nhiều yếu tố hơn, chúng tôi tin rằng mức độ giải thích của mô hình cao hơn.

\section{Tài liệu tham khảo}

Agresti, A. (2007). An introduction to categorical data analysis. Hoboken, NJ: John Wiley \& Sons.

Bộ Tài chính. (2012). Chuẩn mưc kiểm toán VN số 200 - Mục tiêu tổng thể của kiểm toán viên và doanh nghiệp kiểm toán khi thực hiện kiểm toán theo chuẩn mực kiểm toán Việt Nam [Auditing standard VN No. 200 - Overall goal of auditors and auditing firms when performing audits according to Vietnamese Auditing standards]. Hanoi, Vietnam: Nhà xuất bản Tài chính.

Bộ Tài chính. (2012). Chuẩn mưc kiểm toán VN số 220 - Kiểm soát chất luợng hoạt động kiểm toán báo cáo tài chính [Vietnamese auditing standard No. 220 - Quality control of auditing financial statements]. Hanoi, Vietnam: Nhà xuất bản Tài chính.

Bộ Tài Chính. (2012). Chuẩn mưc kiểm toán VN số 315 - Xác định và đánh giá rủi ro có sai sót trọng yếu thông qua hiểu biết về đơn vị được kiểm toán và môi trương của đơn vị [Vietnamese Auditing Standard No. 315 - Identifying and assessing risks of material misstatement through knowledge of the entity and its environment]. Hanoi, Vietnam: Nhà xuất bản Tài chính.

Cenker, W. J., \& Nagy, A. L. (2008). Auditor resignations and auditor industry specialization. Accounting Horizons, 22(3), 279-295. doi:10.2308/acch.2008.22.3.279

Chow, C. W., Ho, J. L., \& Mo, P. L. L. (2006). Toward understanding Chinese auditors' structuring of audit approaches, client acceptance decisions, risk assessment, and stringency of imposed reporting standards. Journal of International Accounting Research, 5(1), 1-23.

Doan, N. T. (2011). Nghiên cưu đánh giá trọng yếu và rủi ro kiểm toán nhằm nâng cao chất luợng hoạt động trong các công ty kiểm toán độc lập Việt Nam [Researching to assess material and audit risks to improve the quality of operations in the independent auditing firms in Vietnam] (Doctoral dissertation). National Economics University, Hanoi, Vietnam. 
Ebaid, I. E.-S. (2011). Corporate governance practices and auditor's client acceptance decision: Empirical evidence from Egypt. Corporate Governance: The International Journal of Business in Society, 11(2), 171-183. doi:10.1108/14720701111121047

Gendron, Y. (2001). The difficult client-acceptance decision in Canadian audit firms: A field investigation. Contemporary Accounting Research, 18(2), 283-310.

Hsieh, Y.-T., \& Lin, C.-J. (2016). Audit firms' client acceptance decisions: Does partner-level industry expertise matter? AUDITING: A Journal of Practice \& Theory, 35(2), 97-120. doi:10.2308/ajpt-51292

Johnstone, K. M. (2000). Client-acceptance decisions: Simultaneous effects of client business risk, audit risk, auditor business risk, and risk adaptation. AUDITING: A Journal of Practice \& Theory, 19(1), 1-25. doi:10.2308/aud.2000.19.1.1

Johnstone, K. M., \& Bedard, J. C. (2003). Risk management in client acceptance decisions. The Accounting Review, 78(4), 1003-1025. doi:10.2308/accr.2003.78.4.1003

Johnstone, K. M., \& Bedard, J. C. (2004). Audit firm portfolio management decisions. Journal of Accounting Research, 42(4), 659-690. doi:10.1111/j.1475-679x.2004.00153.x

Nguyen, T. H. (2013). Rủi ro từ việc không tuân thủ quy trình chấp nhận khách hàng của các công ty kiểm toán [Risks from not complying with the client acceptance process of auditing firms]. Tạp chí Khoa hoc ĐHQGHN, Kinh tế và Kinh doanh, 29(3), 43-52.

Ouertani, I., \& Damak-Ayadi, S. (2012). Auditor engagement decision: An exploratory study in the Tunisian context. Accounting and Management Information Systems, 11(3), 371-390. 